\title{
The Impact of Stieltjes' Work on Continued Fractions and Orthogonal Polynomials
}

\author{
Walter Van Assche ${ }^{1}$ \\ Katholieke Universiteit Leuven
}

\section{Introduction}

The memoir Recherches sur les fractions continues, published posthumously in the Annales de la Faculté des Sciences de Toulouse - a journal of which Stieltjes was one of the first editors - and a great number of other papers by Stieltjes contain a wealth of material that still has a great impact on contemporary research, especially on the theory of orthogonal polynomials. The general theory of orthogonal polynomials really started with the investigations of Chebyshev and Stieltjes. The impact of the work of Chebyshev and his student Markov has already been described by Krein [53]. Here we give an attempt to discuss some of Stieltjes' contributions and the impact on later work. Orthogonal polynomials offer a variety of results and applications. The bibliography [91] up to 1940 consists of 1952 papers by 643 authors. Even now interest in orthogonal polynomials is enormous. One of the reasons is that orthogonal polynomials seem to appear in a great variety of applications. Their use in the numerical approximation of integrals was already pointed out by Gauss and further extended by Christoffel [19] [30] and Stieltjes [95]. The Padé table [73] for the approximation of a function by rational functions is very closely related to continued fractions and Stieltjes' work may be considered as one of the first proofs of convergence in the Padé table [74]. In 1954 Lederman and Reuter [56] and in 1957 Karlin and McGregor [49] showed that the transition probabilities in a birth and death process could be expressed by means of a Stieltjes integral of orthogonal polynomials. Even in pure mathematics there seems to be a natural framework where orthogonal polynomials come into play: representations for certain Lie groups very often are in terms of special functions, in particular orthogonal polynomials (see e.g. Vilenkin [117]). Recently this has also been observed for quantum groups [52]. Discrete orthogonal polynomials have useful applications in the design of association schemes and the proof of nonexistence of perfect codes and orthogonal polynomials on the unit circle have a close connection with digital signal processing. The proceedings of the NATO Advanced Study Institute on "Orthogonal Polynomials and their Applications" (Columbus, Ohio 1989) [69] gives excellent contributions to each of these aspects of orthogonal polynomials and is strongly recommended.

1 The author is a Research Associate of the Belgian National Fund for Scientific Research 
Stieltjes' work has already been discussed by Cosserat [22] shortly after Stieltjes' death in 1894. In these notes we will try to estimate the value of the investigations by Stieltjes a century later. Let me also mention Brezinski's book on the history of continued fractions [10, Chapter 5, Section 5.2.4 on pp. 224-235] where Stieltjes' work on continued fractions is shown in its historic context.

I never quite realized how much work is needed to analyse Stieltjes' work a century after his death. I have spent a lot of time in various libraries and received a lot of help from the librarians. I would also like to thank various colleagues for suggestions, comments and for pointing out omissions and misinterpretations. A sincere word of thanks in particular to Marcel de Bruin, Ted Chihara, Walter Gautschi, Tom Koornwinder and Doron Lubinsky. Of course nothing would have been possible without the help of Gerrit van Dijk: many thanks for having started this whole project.

\section{Stieltjes Continued Fraction}

The object of his main work [105] is the study of the continued fraction

$$
\frac{1}{c_{1} z+\frac{1}{c_{2}+\frac{1}{c_{3} z+\cdots+\frac{1}{c_{2 n}+\frac{1}{c_{2 n+1} z+\cdots}}}},}
$$

which is nowadays known as a Stieltjes continued fraction or $S$-fraction. Stieltjes only considers the case where $c_{k}>0(k=1,2, \ldots)$. In general an $S$-fraction is any continued fraction of the form (1.1) in which all $c_{k}$ are different from zero or any continued fraction which can be obtained from it by an equivalence transformation or change of variable [119, p. 200]. The $S$-fraction (1.1) can be transformed by contraction to a $J$-fraction

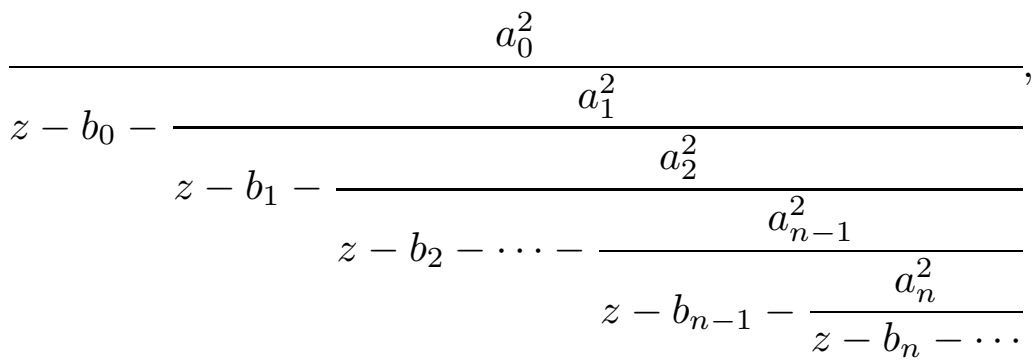

with $a_{0}^{2}=1 / c_{1}, b_{0}=-1 /\left(c_{1} c_{2}\right)$ and

$$
a_{n}^{2}=\frac{1}{c_{2 n-1} c_{2 n}^{2} c_{2 n+1}}, \quad b_{n}=-\frac{1}{c_{2 n} c_{2 n+1}}-\frac{1}{c_{2 n+1} c_{2 n+2}}, \quad k=1,2, \ldots
$$


The positivity of the $c_{k}$, as imposed by Stieltjes, clearly puts some constraints on the coefficients $a_{k}, b_{k}$ e.g., $a_{k}^{2}>0$ and $b_{k}<0$. A $J$-fraction can be regarded as being generated by the sequence of transformations

$$
t_{0}(w)=\frac{1}{w}, \quad t_{k}(w)=z-b_{k-1}-\frac{a_{k}^{2}}{w}, \quad k=1,2, \ldots
$$

The superposition $t_{0}\left(t_{1}\left(\cdots\left(t_{n}(w)\right) \ldots\right)\right)$ for $w=\infty$ is then the $n$-th approximant or $n$-th convergent of the fraction (1.2). This is a rational function of the variable $z$ and we have

$$
t_{0}\left(t_{1}\left(\cdots\left(t_{n}(\infty)\right) \ldots\right)\right)=\frac{1}{a_{1}} \frac{p_{n-1}^{(1)}(z)}{p_{n}(z)},
$$

where both the denominator polynomials $p_{n}(z)(n=0,1,2, \ldots)$ and numerator polynomials $p_{n-1}^{(1)}(z)(n=0,1,2, \ldots)$ are solutions of the three-term recurrence relation

$$
z r_{n}(z)=a_{n+1} r_{n+1}(z)+b_{n} r_{n}(z)+a_{n} r_{n-1}(z), \quad n \geq 0
$$

with initial condition

$$
p_{-1}(z)=0, p_{0}(z)=1, \quad p_{-1}^{(1)}(z)=0, p_{0}^{(1)}(z)=1 .
$$

The convergents of the $S$-fraction are such that the $2 n$-th convergent of (1.1) is equal to the $n$-th convergent of (1.2). If the denominator $p_{n}(z)$ vanishes for at most a finite number of integers $n$ and if $\lim _{n \rightarrow \infty} p_{n-1}^{(1)}(z) / p_{n}(z)=f(z)$ exists, then the $J$-fraction converges to $f(z)$. Stieltjes gave a general theory of $S$-fractions (and consequently of $J$-fractions) with $c_{k}>0(k=1,2, \ldots)$, dealing with questions of convergence and he showed a close connection with asymptotic series in terms of a given sequence of moments (see also the next section).

One of the most important facts in the theory is that the denominators $p_{n}(-x)(n=0,1,2, \ldots)$ form a sequence of orthonormal polynomials on $[0, \infty)$ i.e., there is a positive measure $\mu$ on $[0, \infty)$ such that

$$
\int_{0}^{\infty} p_{n}(-x) p_{m}(-x) d \mu(x)=\delta_{m, n}
$$

The support of the measure $\mu$ is in $[0, \infty)$ precisely because Stieltjes assumes the coefficients $c_{k}$ of the $S$-fraction (1.1) to be positive. Stieltjes showed that such orthogonal polynomials have zeros with interesting properties. He proved that all the zeros of $p_{n}(-x)$ are real, positive and simple; moreover the zeros of $p_{n}(-x)$ interlace with the zeros of $p_{n-1}(-x)$ but also with the zeros of $p_{n-1}^{(1)}(-x)$. The latter property shows that the convergent $p_{n-1}^{(1)}(z) / p_{n}(z)$ is a rational function with $n$ real and negative poles and positive residues. These properties are now quite classical and of great use for numerical quadrature. The property of orthogonality is crucial in these considerations (but Stieltjes 
never uses this terminology). A famous and very important result in the theory of orthogonal polynomials on the real line is the following result:

Theorem. Suppose a system of polynomials satisfies a three-term recurrence relation of the form (1.3) with $a_{k+1}>0$ and $b_{k} \in \mathbb{R}(k=0,1,2, \ldots)$ and initial conditions $r_{-1}(z)=0$ and $r_{0}(z)=1$, then these polynomials are orthonormal in $L^{2}(\mu)$ for some positive measure $\mu$ on the real line.

This theorem is usually called Favard's theorem [25] but it is basically already in Stieltjes' memoir $[105, \S 11]$ for the case of $J$-fractions obtained from contracting an $S$-fraction with positive coefficients: he shows that there is a positive linear functional $S$ such that $S\left(r_{m} r_{n}\right)=0$ whenever $m \neq n$. The only thing that Stieltjes was missing was the Riesz representation theorem which would enable one to express the linear functional $S$ as a Stieltjes integral.

Hilbert's work on quadratic forms in infinitely many variables was much inspired by Stieltjes' work on continued fractions [43, p. 109]: "Die Anwendungen der Theorie der quadratischen Formen mit unendlich vielen Variabeln sind nicht auf die Integralgleichungen beschränkt: es bietet sich nicht minder eine Berührung dieser Theorie mit der schönen Theorie der Kettenbrüche von Stieltjes ...". Stieltjes' theory is full of important ideas. In Chapter V of [105] Stieltjes gives a discussion on the convergence of sums of the form

$$
f_{1}(z)+f_{2}(z)+\cdots+f_{n}(z),
$$

where $f_{i}(z)$ are analytic functions on the open unit disk $C_{R}$ with center at the origin and radius $R$. He proves a result which was later also proved by Giuseppe Vitali in 1903 [118]:

Theorem (Stieltjes-Vitali) Let $f_{n}$ be a sequence of analytic functions on a nonempty connected open set $\Omega$ of the complex plane. If $f_{n}$ is uniformly bounded on compact sets of $\Omega$ and if $f_{n}$ converges on a subset $E \subset \Omega$ that has an accumulation point in $\Omega$, then $f_{n}$ converges uniformly on every compact subset of $\Omega$.

Paul Montel refers to this theorem as Stieltjes' theorem [67] and others refer to it as Vitali's theorem. This result is very convenient in the study of convergence of continued fractions because quite often one is dealing with rational fractions and one may be able to prove convergence on a set $E$ that is far enough away from the poles of the rational fraction. The Stieltjes-Vitali theorem then allows one to extend the asymptotic result to hold everywhere except at the set containing all the poles.

The continued fraction (1.2) was later studied by Van Vleck [116] for $b_{k} \in \mathbb{R}$ and $a_{k}^{2}$ arbitrary positive numbers. The corresponding measure is then not necessarily supported on $[0, \infty)$ and these continued fractions are then closely related to Stieltjes integrals over $(-\infty, \infty)$. The complete extension is due to Hamburger [37]. Van Vleck [115] and Pringsheim [83] [84] have also given an extension to complex coefficients. 
For some good expositions on continued fraction we refer to the books by Perron [78], Wall [119], Jones and Thron [46] and Lorentzen and Waadeland [58].

\section{Moment Problems}

\subsection{The Stieltjes Moment Problem}

In his fundamental work $[105, \S 24]$ Stieltjes introduced the following problem: given an infinite sequence $\mu_{k}(k=0,1,2, \ldots)$, find a distribution of mass (a positive measure $\mu$ ) on the semi-infinite interval $[0, \infty)$ such that

$$
\mu_{k}=\int_{0}^{\infty} x^{k} d \mu(x), \quad k=0,1,2, \ldots
$$

Of course such a measure will not always exist for any sequence $\mu_{k}$ and if such a measure exists, then it need not be unique. The Stieltjes moment problem therefore has two parts

1. find necessary and/or sufficient conditions for the existence of a solution of the moment problem on $[0, \infty)$,

2. find necessary and/or sufficient conditions for the uniqueness of the solution of the moment problem on $[0, \infty)$.

Chebyshev had previously investigated integrals and sums of the form

$$
\int_{-\infty}^{\infty} \frac{w(t)}{x-t} d t, \quad \sum_{i=0}^{\infty} \frac{w_{i}}{x-x_{i}}
$$

where $w(t)$ is a positive weight function and $w_{i}$ are positive weights. Stieltjes integrals cover both cases and give a unified approach to the theory. Chebyshev did not investigate a moment problem, but was interested when a given sequence of moments determines the function $w(x)$ or the weights $w_{i}$ uniquely. His work and the work of his student Markov is very relevant, but Stieltjes apparently was unaware of it. See Krein [53] for some history related to the work of Chebyshev and Markov. Nevertheless Stieltjes' introduction of the moment problem is still regarded as an important mathematical achievement. The reason for the introduction of this moment problem is a close connection between $S$-fractions or $J$-fractions and infinite series. If we make a formal expansion of the function

$$
S(\mu ; x)=\int \frac{d \mu(t)}{x+t}
$$

which is known as the Stieltjes transform of the measure $\mu$, then we find

$$
\int \frac{d \mu(t)}{x+t} \sim \sum_{k=0}^{\infty}(-1)^{k} \frac{\mu_{k}}{x^{k+1}} .
$$


This series does not always converge and should be considered as an asymptotic expansion. On the other hand one can expand the function $S(\mu ; x)$ also into a continued fraction of the form (1.1) or (1.2). The $n$-th approximant of the $J$-fraction has the property that the first $2 n$ terms in the expansion

$$
\frac{1}{a_{1}} \frac{p_{n-1}^{(1)}(x)}{p_{n}(x)} \sim \sum_{k=0}^{\infty}(-1)^{k} \frac{m_{k}}{x^{k+1}}
$$

agree with those of the expansion of $S(\mu ; x)$ i.e., $m_{k}=\mu_{k}$ for $k=0,1, \ldots, 2 n-$ 1. This rational function is therefore a (diagonal) Padé approximant for $S(\mu ; x)$. If the infinite series is given, then the continued fraction is completely known whenever the measure $\mu$ is known, provided the continued fraction converges.

Stieltjes gave necessary and sufficient conditions for the existence of a solution of the Stieltjes moment problem:

Theorem. If the Hankel determinants satisfy

$$
\left|\begin{array}{cccc}
\mu_{0} & \mu_{1} & \cdots & \mu_{n} \\
\mu_{1} & \mu_{2} & \cdots & \mu_{n+1} \\
\vdots & \vdots & \cdots & \vdots \\
\mu_{n} & \mu_{n+1} & \cdots & \mu_{2 n}
\end{array}\right|>0, \quad n \in \mathbb{N}
$$

and

$$
\left|\begin{array}{cccc}
\mu_{1} & \mu_{2} & \cdots & \mu_{n+1} \\
\mu_{2} & \mu_{3} & \cdots & \mu_{n+2} \\
\vdots & \vdots & \cdots & \vdots \\
\mu_{n+1} & \mu_{n+2} & \cdots & \mu_{2 n+1}
\end{array}\right|>0, \quad n \in \mathbb{N},
$$

then there exists a solution of the Stieltjes moment problem.

If the moment problem has a unique solution then the moment problem is determinate. If there exist at least two solutions then the moment problem is indeterminate. Other terminology is also in use: determined/indetermined and determined/undetermined. Any convex combination of two solutions is another solution, hence in case of an indeterminate moment problem there will always be an infinite number of solutions. Stieltjes gave explicit examples of indeterminate moment problems (see also Section 5.3) and he showed that a moment problem is determinate if and only if the corresponding continued fraction (1.1) converges for every $z$ in the complex plane, except for $z$ real and negative. A necessary and sufficient condition for a determinate moment problem is the divergence of the series $\sum_{n=1}^{\infty} c_{n}$ where $c_{n}$ are the coefficients of the $S$-fraction (1.1). In case of an indeterminate moment problem Stieltjes constructs two solutions as follows: let $P_{n}(z) / Q_{n}(z)$ be the $n$-th convergent of the continued fraction (1.1), then the limits 


$$
\begin{aligned}
& \lim _{n \rightarrow \infty} P_{2 n}(z)=p(z), \quad \lim _{n \rightarrow \infty} P_{2 n+1}(z)=p_{1}(z), \\
& \lim _{n \rightarrow \infty} Q_{2 n}(z)=q(z), \quad \lim _{n \rightarrow \infty} Q_{2 n+1}(z)=q_{1}(z),
\end{aligned}
$$

exist, where $p, p_{1}, q, q_{1}$ are entire functions satisfying

$$
q(z) p_{1}(z)-q_{1}(z) p(z)=1 .
$$

Stieltjes then shows that

$$
\frac{p(z)}{q(z)}=\sum_{k=1}^{\infty} \frac{r_{k}}{z+x_{k}}, \quad \frac{p_{1}(z)}{q_{1}(z)}=\frac{s_{0}}{z}+\sum_{k=1}^{\infty} \frac{s_{k}}{z+y_{k}} .
$$

The poles $x_{k}, y_{k}(k=1,2, \ldots)$ are all real and positive and the residues $r_{k}, s_{k}$ are all positive: this follows because the zeros of the numerator polynomials interlace with the zeros of the numerator polynomials and because all these zeros are real and negative. These limits can thus be expressed as a Stieltjes integral

$$
\frac{p(z)}{q(z)}=\int_{0}^{\infty} \frac{d \mu(t)}{z+t}, \quad \frac{p_{1}(z)}{q_{1}(z)}=\int_{0}^{\infty} \frac{d \mu_{1}(t)}{z+t},
$$

and both $\mu$ and $\mu_{1}$ are solutions of the moment problem with remarkable extremal properties. This is one instance where it is clear why Stieltjes introduced the concept of a Stieltjes integral.

Not much work on the Stieltjes moment problem was done after Stieltjes' death. One exception is G. H. Hardy [38] who considered the moments of a weight function $w(x)$ on $[0, \infty)$ with restricted behaviour at infinity:

$$
\int_{0}^{\infty} w(x) e^{k \sqrt{x}} d x<\infty
$$

for a positive value of $k$. He shows that the Stieltjes moment problem is then always determinate and constructs the density from the series

$$
\sum_{n=0}^{\infty} \frac{\mu_{n}(-x)^{n}}{(2 n) !}
$$

Hardy's proof avoids the use of continued fractions.

\subsection{Other Moment Problems}

Nothing new happened until 1920 when Hamburger [37] extended Stieltjes' moment problem by allowing the solution to be a measure on the whole real line instead of the positive interval $[0, \infty)$. The extension seems straightforward but the analysis is more complicated because the coefficients of the continued fraction (1.1) may become negative or vanish. Hamburger showed, using continued fraction techniques, that a necessary and sufficient condition for the existence of a solution of the Hamburger moment problem is the positivity of the Hankel determinants (2.1). He also shows that a Hamburger moment 
problem may be indeterminate while the Stieltjes moment problem with the same moments is determinate.

Nevanlinna [70] introduced techniques of modern function theory to investigate moment problems without using continued fractions. His work is important because of the notion of extremal solutions, which were first studied by him. M. Riesz [85] [86] gave a close connection between the density of polynomials in $L^{2}$-spaces and moment problems:

Theorem. Let $\mu$ be a positive measure on $(-\infty, \infty)$. If the Hamburger moment problem for $\mu_{k}=\int x^{k} d \mu(x)(k=0,1,2, \ldots)$ is determinate, then polynomials are dense in $L^{2}(\mu)$. If the Hamburger moment problem is indeterminate then the polynomials are dense in $L^{2}(\mu)$ if and only if $\mu$ is a Nevanlinna extremal measure.

Berg and Thill [9] have recently pointed out that this connection is not any longer valid in higher dimensions by showing that there exist rotation invariant measures $\mu$ on $\mathbb{R}^{d}, d>1$ for which the moment problem is determinate but for which polynomials are not dense in $L^{2}(\mu)$.

In 1923 Hausdorff [39] studied the moment problem for measures on a finite interval $[a, b]$. The Hausdorff moment problem is always determinate and conditions for the existence of a solution can be given in terms of completely monotonic sequences. The moment problem is closely related to quadratic forms of infinitely many variables and operators in Hilbert space, as became clear from the work of Carleman [12] [13] and Stone [107]. Carleman established the following sufficient condition for a determinate moment problem:

$$
\sum_{k=1}^{\infty} \mu_{2 k}^{-1 / 2 k}=\infty
$$

This is still the most general sufficient condition. Karlin and his collaborators [50] [51] have approached the moment problem through the geometry of convex sets and have shown that many results can be interpreted in this geometrical setting. Let me mention here that one can find excellent treatments of the moment problem in the monograph of Shohat and Tamarkin [92] and the book of Akhiezer [3]. Also of interest is the monograph by Krein and Nudelman [54].

\subsection{Recent Extensions of the Moment Problem}

The most recent extension of the moment problem is to consider a doubly infinite sequence $\mu_{n}(n \in \mathbb{Z})$ and to find a positive measure $\mu$ on $(-\infty, \infty)$ such that

$$
\mu_{n}=\int_{-\infty}^{\infty} x^{n} d \mu(x), \quad n \in \mathbb{Z} .
$$

Such a moment problem is known as a strong moment problem. The strong Stieltjes moment problem was posed and solved by Jones, Thron and Waadeland in 1980 [48] and again the solution is given in terms of the positivity of 
certain Hankel determinants. These authors again use continued fractions, but instead of the $S$ - and $J$-fractions encountered by Stieltjes and Hamburger, one deals with another kind of fraction known as a $T$-fraction. The strong Hamburger moment problem was handled by Jones, Thron and Njåstad in 1984 [47]. Njåstad [71] gave another extension, known as the extended moment problem: given $p$ sequences $\mu_{n}^{(k)}(n=1,2, \ldots ; 1 \leq k \leq p)$ and $p$ real numbers $a_{1}, a_{2}, \ldots, a_{p}$, does there exist a positive measure $\mu$ on the real line such that

$$
\mu_{n}^{(k)}=\int_{-\infty}^{\infty} \frac{d \mu(t)}{\left(t-a_{k}\right)^{n}}, \quad 1 \leq k \leq p, n \in \mathbb{N} ?
$$

The solution is again given in terms of positive definiteness of a certain functional. Orthogonal polynomials play an important role in the Stieltjes and Hamburger moment problem; for the strong moment problem a similar important role is played by orthogonal Laurent polynomials and for the extended moment problem one deals with orthogonal rational functions. The first place where orthogonal Laurent polynomials are considered seems to be a paper by Pastro [75], where an explicit example of the orthogonal Laurent polynomials with respect to the Stieltjes-Wigert weight appears (see $\S 5.3$ for this weight).

\section{Electrostatic Interpretation of Zeros}

Stieltjes gave a very interesting interpretation of the zeros of Jacobi, Laguerre and Hermite polynomials in terms of a problem of electrostatic equilibrium. Suppose $n$ unit charges at points $x_{1}, x_{2}, \ldots, x_{n}$ are distributed in the (possibly infinite) interval $(a, b)$. The expression

$$
D\left(x_{1}, x_{2}, \ldots, x_{n}\right)=\prod_{1 \leq i<j \leq n}\left|x_{i}-x_{j}\right|
$$

is known as the discriminant of $x_{1}, x_{2}, \ldots, x_{n}$. If the charges repell each other according to the law of logaritmic potential, then

$$
-\log D\left(x_{1}, x_{2}, \ldots, x_{n}\right)=\sum_{1 \leq i<j \leq n} \log \frac{1}{\left|x_{i}-x_{j}\right|}
$$

is the energy of the system of electrostatic charges and the minimum of this expression gives the electrostatic equilibrium. The points $x_{1}, x_{2}, \ldots, x_{n}$ where the minimum is obtained are the places where the charges will settle down. Stieltjes observed that these points are closely related to zeros of classical orthogonal polynomials.

\subsection{Jacobi Polynomials}

Suppose the $n$ unit charges are distributed in $[-1,1]$ and that we add two extra charges at the endpoints, a charge $p>0$ at +1 and a charge $q>0$ at 
-1 . Each of the unit charges interacts with the charges at \pm 1 and therefore the electrostatic energy becomes

$$
L=-\log D_{n}\left(x_{1}, x_{2}, \ldots, x_{n}\right)+p \sum_{i=1}^{n} \log \frac{1}{\left|1-x_{i}\right|}+q \sum_{i=1}^{n} \log \frac{1}{\left|1+x_{i}\right|} .
$$

Stieltjes then proved the following result [97] [98] [100]

Theorem. The expression (3.1) becomes a minimum when $x_{1}, x_{2}, \ldots, x_{n}$ are the zeros of the Jacobi polynomial $P_{n}^{(2 p-1,2 q-1)}(x)$.

Proof. It is clear that for the minimum all the $x_{i}$ are distinct and different from \pm 1 . For a minimum we need $\partial L / \partial x_{k}=0(1 \leq k \leq n)$ so that we have the system of equations

$$
\sum_{\substack{i=1 \\ i \neq k}}^{n} \frac{1}{x_{i}-x_{k}}-\frac{p}{x_{k}-1}-\frac{q}{x_{k}+1}=0, \quad 1 \leq k \leq n .
$$

If we introduce the polynomial

$$
p_{n}(x)=\left(x-x_{1}\right)\left(x-x_{2}\right) \cdots\left(x-x_{n}\right),
$$

then this is equivalent with

$$
\frac{1}{2} \frac{p_{n}^{\prime \prime}\left(x_{k}\right)}{p_{n}^{\prime}\left(x_{k}\right)}+\frac{p}{x_{k}-1}+\frac{q}{x_{k}+1}=0, \quad 1 \leq k \leq n .
$$

This means that the polynomial

$$
\left(1-x^{2}\right) p_{n}^{\prime \prime}(x)+2[q-p-(p+q) x] p_{n}^{\prime}(x)
$$

vanishes at the points $x_{k}$ and since this polynomial is of degree $n$ it must be a multiple of $p_{n}(x)$. The factor is easily obtained by equating the coefficient of $x^{n}$ and we have

$$
\left(1-x^{2}\right) p_{n}^{\prime \prime}(x)+2[q-p-(p+q) x] p_{n}^{\prime}(x)=-n[n+2(p+q)-1] p_{n}(x),
$$

which is the differential equation for the Jacobi polynomial $P_{n}^{(2 p-1,2 q-1)}(x) / c_{n}$, where $c_{n}$ is the leading coefficient of the Jacobi polynomial.

Stieltjes also found the minimum value. Hilbert [42] also computed the minimum value and Schur [89] treated the case $p=q=0$ in detail. Schur's paper then led Fekete [26] to define the transfinite diameter of a compact set $K$ (with infinitely many points) in the complex plane. Take $n$ points $z_{i} \in K$ $(i=1,2, \ldots, n)$, and put

$$
d_{n}=\max _{z_{i} \in K} D\left(z_{1}, \ldots, z_{n}\right)^{1 /\left(\begin{array}{c}
n \\
2
\end{array}\right)},
$$

then $d_{n}$ is a decreasing and positive sequence [26] [111, Thm. III.21 on p. 71]. The limit of this sequence is the transfinite diameter of $K$ and is an important 
quantity in logarithmic potential theory (see §3.4). The transfinite diameter thus comes directly from Stieltjes' work.

Consider the function

$$
\left(\prod_{i=1}^{n}\left|1-x_{i}\right|^{x-1}\left|1+x_{i}\right|^{y-1}\right) D_{n}\left(x_{1}, \ldots, x_{n}\right),
$$

then Stieltjes' electrostatic interpretation gives the $L_{[-1,1]^{n}}^{\infty}$-norm of this function. The $L_{[-1,1]^{n}}^{p}$-norm of this function is also very famous and is known as Selberg's beta integral [90]. Actually Selberg evaluated a multiple integral over $[0,1]^{n}$ :

$$
\begin{gathered}
\int_{0}^{1} \ldots \int_{0}^{1} D_{n}\left(t_{1}, \ldots, t_{n}\right)^{2 z}\left(\prod_{i=1}^{n} t_{i}^{x-1}\left(1-t_{i}\right)^{y-1}\right) d t_{1} \ldots d t_{n} \\
=\prod_{j=1}^{n} \frac{\Gamma(x+(j-1) z) \Gamma(y+(j-1) z) \Gamma(j z+1)}{\Gamma(x+y+(n+j-2) z) \Gamma(z+1)},
\end{gathered}
$$

but this integral can easily be transformed to an integral over $[-1,1]^{n}$ which by an appropriate choice of the parameters $z, x, y$ becomes the desired $L_{[-1,1]^{n^{-}}}^{p}$ norm. This multiple integral has many important applications e.g., in the statistical theory of high energy levels (Mehta [64]) but also in the algebraic theory of root systems (Macdonald [60]). Aomoto [6] gave an elementary evaluation of Selberg's integral and Gustafson [35] computed some $q$-extensions. Selberg's work was not inspired by Stieltjes, but it is directly related to it.

\subsection{Laguerre and Hermite Polynomials}

A similar interpretation exists for the zeros of Laguerre and Hermite polynomials. Suppose the $n$ unit charges are distributed in $[0, \infty)$ and that we add one extra charge $p>0$ at the origin. In order to prevent the charges from moving to $\infty$ we add the extra condition that the centroid satisfies

$$
\frac{1}{n} \sum_{k=1}^{n} x_{i} \leq K
$$

with $K$ a positive number. The energy now is given by the expression

$$
L=-\log D_{n}\left(x_{1}, \ldots, x_{n}\right)+p \sum_{k=1}^{n} \log \frac{1}{x_{k}} .
$$

Theorem. The expression (3.3) with the constraint (3.2) has a minimum when $x_{1}, x_{2}, \ldots, x_{n}$ are the zeros of the Laguerre polynomial $L_{n}^{(2 p-1)}\left(c_{n} x\right)$, where $c_{n}=(n+2 p-1) / K$.

If the $n$ unit charges are on $(-\infty, \infty)$ and if the moment of inertia satisfies 


$$
\frac{1}{n} \sum_{k=1}^{n} x_{k}^{2} \leq L,
$$

then

Theorem. The expression $-\log D_{n}\left(x_{1}, x_{2}, \ldots, x_{n}\right)$ with constraint (3.4) becomes minimal when $x_{1}, x_{2}, \ldots, x_{n}$ are the zeros of the Hermite polynomial $H_{n}\left(d_{n} x\right)$, where $d_{n}=\sqrt{(n-1) / 2 L}$.

The proof of both statements is similar to the proof for the Jacobi case, except that now we use a Lagrange multiplier to find the constrained minimum. Mehta's book on Random Matrices [64] gives an alternative way to prove the results for Laguerre and Hermite polynomials.

In 1945 Siegel [93] reproved the theorem for Laguerre polynomials and applied it to improve the arithmetic-geometric mean inequality and to find better bounds on algebraic integers. Siegels seems not to have been aware of Stieltjes' work, but started from Schur's work [89].

\subsection{Extensions}

In [99] Stieltjes generalizes this idea to polynomial solutions of the differential equation

$$
A(x) y^{\prime \prime}+2 B(x) y^{\prime}+C(x) y=0,
$$

where $A, B$ and $C$ are polynomials of degree respectively $p+1, p$ and $p-1$. Such a differential equation is known as a Lamé equation in algebraic form. In 1878 Heine asserted that when $A$ and $B$ are given, there are in general exactly $\left(\begin{array}{c}n+p-1 \\ n\end{array}\right)$ polynomials $C$ such that the differential equation has a solution which is a polynomial of degree $n$. Stieltjes assumes that

$$
\frac{B(x)}{A(x)}=\sum_{k=0}^{p} \frac{r_{k}}{x-a_{k}},
$$

with $r_{k}>0$ and $a_{k} \in \mathbb{R}$. One can then put charges $r_{k}$ at the points $a_{k}$ and $n$ unit charges at $n$ points $x_{1}, x_{2}, \ldots, x_{n}$ on the real line. Stieltjes then shows that there are exactly $\left(\begin{array}{c}n+p-1 \\ n\end{array}\right)$ positions of electrostatic equilibrium, each corresponding to one particular distribution of the $n$ charges in the $p$ intervals $\left[a_{k}, a_{k+1}\right](0 \leq k<p)$, and these charges are then at the points $x_{1}, x_{2}, \ldots, x_{n}$ which are the $n$ zeros of the polynomial solution of the differential equation. This result is now known as the Heine-Stieltjes theorem [110, Theorem 6.8 on p. 151]. The conditions imposed by Stieltjes have been weakened by Van Vleck [114] and Pólya [79]. Pólya allowed the zeros of $A$ to be complex and showed that the zeros of the polynomial solution of the differential equation will all belong to the convex hull of $\left\{a_{0}, \ldots, a_{p}\right\}$. The location of the zeros of the polynomial solution is still under investigation now and interesting results and applications to certain problems in physics and fluid mechanics are discussed in [4] [5] [123]. 
Recently Forrester and Rogers [27] and Hendriksen and van Rossum [40] have allowed the $n$ unit charges to move into the complex plane. Forrester and Rogers consider a system of $2 n$ particles of unit charge confined to a circle in the complex plane, say at the points $e^{i \theta_{j}}$ and $e^{-i \theta_{j}}(1 \leq j \leq n)$. At $\theta=0$ (i.e., at the point $z=1$ ) a particle of charge $q$ is fixed and at $\theta=\pi(z=-1)$ a particle of charge $p$. The energy of the system is now given by

$$
L=-q \sum_{k=1}^{2 n} \log \left|1-e^{i \theta_{k}}\right|-p \sum_{k=1}^{2 n} \log \left|1+e^{i \theta_{k}}\right|-\sum_{1 \leq k<j \leq 2 n} \log \left|e^{i \theta_{k}}-e^{i \theta_{j}}\right|,
$$

where

$$
0<\theta_{j}<\pi, \quad \theta_{j}+\theta_{n+j}=2 \pi, \quad 1 \leq j \leq n .
$$

Theorem (Forrester and Rogers) The minimum of L given in (3.6) subject to the constraints (3.7) occurs when $\theta_{j}$ are the zeros of the trigonometric Jacobi polynomial $P_{n}^{\left(q-\frac{1}{2}, p-\frac{1}{2}\right)}(\cos \theta)$.

Forrester and Rogers also consider crystal lattice structures in which $n 2^{m}$ particles of unit charge and $2^{m}$ particles of charge $q$ are distributed on the unit circle, with one of the $q$ charges fixed at $\theta=0$. If one requires that between every two $q$ charges there are $n$ unit charges then the equilibrium position of the $n 2^{m}$ particles of unit charge occurs at the zeros of the Jacobi polynomial $P_{n / 2}^{\left(\frac{q-1}{2},-\frac{1}{2}\right)}\left(\cos 2^{m} \theta\right)$ when $n$ is even and at the zeros of $P_{(n-1) / 2}^{\left(\frac{q-1}{2}, \frac{1}{2}\right)}\left(\cos 2^{m} \theta\right)$ when $n$ is odd. The equilibrium position of the $2^{m}-1$ particles of charge $q$ occurs at $\theta_{k}=\frac{2 \pi k}{2^{m}}\left(1 \leq k<2^{m}\right)$.

Hendriksen and van Rossum [40] have considered situations where other special polynomials come into play. Suppose $a>0$ and that there is a charge $(a+1) / 2$ at the origin and a charge $(c-a) / 2$ at the point $1 / a$. If $a \rightarrow \infty$ one obtains a generalized dipole at the origin. Suppose now that there are $n$ unit charges at points $z_{1}, z_{2}, \ldots, z_{n}$ in the complex plane, then the electrostatic equilibrium in this generalized dipole field is obtained when $z_{1}, \ldots, z_{n}$ are the zeros of the Bessel polynomial ${ }_{2} F_{0}(-n, c+n ; x)$. Similar results can be obtained on so-called $m$-stars

$$
S_{m}=\left\{z \in \mathbb{C}: z=\rho e^{\frac{2 \pi k}{m} i}, 0 \leq \rho \leq r, k=0,1,2, \ldots, m-1\right\} .
$$

Suppose that positive charges $q$ are placed at the endpoints $\rho=r$ of $S_{m}$ and a charge $p \geq 0$ is placed at the origin. If the points $z_{1}, \ldots, z_{n}(n>m)$ in the complex plane all have a unit charge, then the electrostatic equilibrium (assuming rotational symmetry) is obtained by choosing $z_{1}, \ldots, z_{n}$ to be the zeros of the polynomial $f_{n}$ of degree $n$ that is a solution of the differential equation

$$
\left(r^{m}-z^{m}\right) z y^{\prime \prime}-2\left[(p+q m) z^{m}-p r^{m}\right] y^{\prime}=-n(n-1+2 p+2 q m) z^{m-1} y .
$$

For particular choices of the parameters $p, r, m$ one then obtains well known (orthogonal) polynomials. 


\subsection{Logarithmic Potential Theory}

Suppose that we normalize the electrostatic problem on $[-1,1]$ in such a way that the total charge is equal to 1 . The $n$ charges then are equal to $1 /(n+p+q)$ and the charges at 1 and -1 become respectively $p /(n+p+q)$ and $q /(n+p+q)$. What happens if the number of particles $n$ increases? Clearly the charges at the endpoints \pm 1 become negligible compared to the total charge of the particles inside $[-1,1]$. This is the only place where $p$ and $q$ affect the distribution of the zeros, therefore it follows that the asymptotic distribution of the charges in $(-1,1)$ i.e., the asymptotic distribution of the zeros of Jacobi polynomials $P_{n}^{(2 p-1,2 q-1)}(x)$, is independent of $p$ and $q$. By taking $p=q=1 / 4$ we deal with Chebyshev polynomials of the first kind $T_{n}(x)$ with zeros $\cos \frac{(2 j-1) \pi}{2 n}$ : $1 \leq j \leq n\}$. Let $N_{n}(a, b)$ be the number of zeros of $T_{n}(x)$ in $[a, b]$, then

$$
\begin{aligned}
\frac{N_{n}(a, b)}{n} & =\sum_{a \leq \cos \frac{(2 j-1) \pi}{2 n} \leq b} \frac{1}{n} \\
& =\int_{a \leq \cos t \pi \leq b} 1 d t+o(1) \\
& =\frac{1}{\pi} \int_{a}^{b} \frac{d x}{\sqrt{1-x^{2}}}+o(1) .
\end{aligned}
$$

Therefore the asymptotic distribution of the zeros of Jacobi polynomials is given by the arcsin distribution and the relative number of zeros in $[a, b]$ is

$$
\frac{1}{\pi} \int_{a}^{b} \frac{d x}{\sqrt{1-x^{2}}}=\frac{1}{\pi}(\arcsin b-\arcsin a) .
$$

The surprising thing is that this is valid not only for Jacobi polynomials but for a very large class of orthogonal polynomials on $[-1,1]$. The arcsin distribution is actually an extremal measure in logarithmic potential theory. Widom [120] [121] and Ullman [112] were probably the first to connect logarithmic potential theory and general orthogonal polynomials, even though some aspects such as the transfinite diameter and conformal mappings had already appeared in earlier work by Szegö [110, Chapter XVI]. Let $K$ be a compact set in $\mathbb{C}$ and denote by $\Omega_{K}$ be the set of all probability measures on $K$. Define for $\mu \in \Omega_{K}$ the logarithmic energy by

$$
I(\mu)=\int_{K} \int_{K} \log \frac{1}{|x-y|} d \mu(x) d \mu(y),
$$

then there exists a unique measure $\mu_{K} \in \Omega_{K}$ such that

$$
I\left(\mu_{K}\right)=\min _{\mu \in \Omega_{K}} I(\mu),
$$

and this measure is the equilibrium measure (see e.g. [111]). When $K=[-1,1]$ then the equilibrium measure turns out to be the arcsin measure. The capacity of the compact set $K$ is given by 


$$
\operatorname{cap}(K)=e^{-I\left(\mu_{K}\right)},
$$

and the capacity of a Borel set $B \in \mathcal{B}$ is defined as

$$
\operatorname{cap}(B)=\sup _{K \subset B, K \text { compact }} \operatorname{cap}(K)
$$

(the capacity of $B$ is allowed to be $\infty$ ). Szego" [108] showed that the capacity of a compact set $K$ is the same as the transfinite diameter of this set, which we defined earlier. The following result concerning the asymptotic distribution of zeros of orthogonal polynomials is known (see e.g. [94]):

Theorem. Let $\mu$ be a probability measure on a compact set $K \subset \mathbb{R}$ such that

$$
\inf _{\mu(B)=1, B \in \mathcal{B}} \operatorname{cap}(B)=\operatorname{cap}(K),
$$

where $\mathcal{B}$ are the Borel subsets of $K$, and suppose that $x_{k, n}(1 \leq k \leq n)$ are the zeros of the orthogonal polynomial of degree $n$ for the measure $\mu$. Then

$$
\lim _{n \rightarrow \infty} \frac{1}{n} \sum_{k=1}^{n} f\left(x_{k, n}\right)=\int_{K} f(t) d \mu_{K}(t)
$$

holds for every continuous function $f$ on $K$.

When $K=[-1,1]$ then the conditions hold when $\mu$ is absolutely continuous on $(-1,1)$ with $\mu^{\prime}(x)>0$ almost everywhere (in Lebesgue sense). This includes all Jacobi weights. A very detailed account of logarithmic potential theory and orthogonal polynomials can be found in a forthcoming book by $\mathrm{H}$. Stahl and V. Totik [94].

There is a similar generalization of the electrostatic interpretation of the zeros of Laguerre and Hermite polynomials. This time we need to introduce the energy of a measure in an external field $f$. If $K$ is a closed set in the complex plane $\mathbb{C}$ and if the field $f: K \rightarrow[0, \infty)$ is admissible i.e.,

1. $f$ is upper semi-continuous,

2. the set $\{z \in K: f(z)>0\}$ has positive capacity ( $\infty$ is allowed),

3. if $K$ is unbounded then $z f(z) \rightarrow 0$ as $|z| \rightarrow \infty(z \in K)$,

then we define the energy integral in the field $f$ as

$$
I_{f}(\mu)=\int_{K} \int_{K} \log \frac{1}{|x-y|} d \mu(x) d \mu(y)-2 \int_{K} \log f(x) d \mu(x) .
$$

Again there exists a unique measure $\mu_{f}$ such that

$$
I_{f}\left(\mu_{f}\right)=\min _{\mu \in \Omega_{K}} I_{f}(\mu),
$$

and this measure is the equilibrium measure in the external field $f$ [34] [65]. The following result generalizes the electrostatic interpretation of the zeros of Hermite polynomials (see e.g. [34]): 
Theorem. Suppose that $x_{k, n}(1 \leq k \leq n)$ are the zeros of the $n$-th degree orthogonal polynomial with weight function $w(x)$ on $(-\infty, \infty)$. Suppose that there exists a positive and increasing sequence $c_{n}$ such that

$$
\lim _{n \rightarrow \infty} w\left(c_{n} x\right)^{1 / n}=f(x), \quad x \in \mathbb{R},
$$

uniformly on every closed interval, with $f$ an admissible field, then

$$
\lim _{n \rightarrow \infty} \frac{1}{n} \sum_{k=1}^{n} g\left(\frac{x_{k, n}}{c_{n}}\right)=\int g(x) d \mu_{f}(x),
$$

for every bounded and continuous function $g$.

Again the asymptotic distribution of the (contracted) zeros of orthogonal polynomials does not depend on the exact magnitude of the weight function $w$, but only on the asymptotic behaviour given in (3.8). When $w(x)=e^{-|x|^{\alpha}}$ - the so-called Freud weights - then one can take $c_{n}=c(\alpha) n^{1 / \alpha}$ with

$$
c(\alpha)=\left(\frac{\sqrt{\pi} \Gamma\left(\frac{\alpha+1}{2}\right)}{\Gamma\left(\frac{\alpha}{2}\right)}\right)^{1 / \alpha},
$$

to find that $f(x)=e^{-|c(\alpha) x|^{\alpha}}$. The corresponding equilibrium measure $\mu_{f}$ has support on $[-1,1]$ and is absolutely continuous with weight function

$$
\mu_{f}^{\prime}(t)=\frac{1}{\pi} \int_{|t|}^{1} \frac{d y^{\alpha}}{\sqrt{y^{2}-t^{2}}}, \quad-1 \leq t \leq 1 .
$$

This is now known as the Nevai-Ullman weight. Notice that the logarithm of the external field is the mathematical counterpart of the constraints (3.2) and (3.4) for the Laguerre and Hermite polynomials.

The fascinating aspects of logaritmic potential theory and zeros of orthogonal polynomials are very much inspired by Stieltjes' observation that the zeros of Jacobi, Laguerre and Hermite polynomials actually solve an equilibrium problem in electrostatics.

\section{Markov-Stieltjes Inequalities}

In his paper [95] Stieltjes generalized the Gaussian quadrature formula, which Gauss gave for the zeros of Legendre polynomials, to general weight functions on an interval $[a, b]$. E. B. Christoffel had given this generalization already seven years earlier [19] [30], but Stieltjes' paper is the first that makes a study of the convergence of the quadrature formula. The Gaussian quadrature formula approximates the integral

$$
\int_{a}^{b} \pi(x) d \mu(x)
$$


by appropriately summing $n$ function evaluations

$$
\sum_{j=1}^{n} \lambda_{j, n} \pi\left(x_{j, n}\right)
$$

This formula has maximal accuracy $2 n-1$ i.e., the sum is equal to the integral for all polynomials of degree at most $2 n-1$, when the quadrature nodes are the zeros $x_{j, n}(1 \leq j \leq n)$ of the orthogonal polynomial $p_{n}(x)$ of degree $n$ with orthogonality measure $\mu$, and the quadrature weights $\lambda_{j, n}(1 \leq j \leq n)$ are given by

$$
\lambda_{j, n}=\frac{-1}{a_{n+1} p_{n}^{\prime}\left(x_{j, n}\right) p_{n+1}\left(x_{j, n}\right)}=\frac{1}{a_{n} p_{n}^{\prime}\left(x_{j, n}\right) p_{n-1}\left(x_{j, n}\right)},
$$

where we have used the recurrence relation (1.3). These weights are known as the Christoffel numbers and have important properties. One of the most important properties is their positivity, which follows easily from

$$
\lambda_{j, n}=\left\{\sum_{k=0}^{n-1} p_{k}^{2}\left(x_{j, n}\right)\right\}^{-1} .
$$

Stieltjes gives another remarkable property, namely

$$
\sum_{j=1}^{k-1} \lambda_{j, n}<\int_{a}^{x_{k, n}} d \mu(x)=\mu\left[a, x_{k, n}\right) \leq \mu\left[a, x_{k, n}\right]<\sum_{j=1}^{k} \lambda_{j, n} .
$$

Stieltjes was unaware that Chebyshev had already conjectured these inequalities in [15] and that Chebyshev's student A. A. Markov had proved them in [62]. Markov's paper appeared in 1884, the same year as Stieltjes' paper [95], but in [96] Stieltjes kindly acknowledges Markov to be the first to prove the inequalities. He says however that his proof is independent of Markov's proof since [95] was submitted in May 1884 whereas Markov's paper arrived at the library in September 1884. Szegő [110] gives three proofs of (4.3), combining the proofs of Stieltjes and Markov. The inequalities (4.3) are nowadays known as the Markov-Stieltjes inequalities. A related set of inequalities was proved by K. Possé [80] [81]. If $f:(a, b) \rightarrow \mathbb{R}$ is such that $f^{(j)}(x) \geq 0$ for every $x \in\left(a, x_{k, n}\right](j=0,1,2, \ldots, 2 n-1)$ then

$$
\sum_{j=1}^{k-1} \lambda_{j, n} f\left(x_{j, n}\right) \leq \int_{a}^{x_{k, n}} f(x) d \mu(x) \leq \sum_{j=1}^{k} \lambda_{j, n} f\left(x_{j, n}\right) .
$$

Stieltjes [96] [106] also gives other inequalities for the Christoffel numbers e.g.,

$$
\sum_{j=1}^{k-1} \lambda_{j, n}<\sum_{j=1}^{k} \lambda_{j, n+1}<\sum_{j=1}^{k} \lambda_{j, n}
$$


Stieltjes used the Markov-Stieltjes inequalities to show that the sum (4.2) takes the form of a Riemann-Stieltjes sum for the integral (4.1), which makes Stieltjes the first to prove convergence of Gaussian quadrature for continuous functions. If $z \in \mathbb{C} \backslash[a, b]$ then the function $f(x)=1 /(z-x)$ is continuous on $[a, b]$ and hence the Gaussian quadrature applied to $f$ converges to the Stieltjes transform of the orthogonality measure $\mu$. The Gaussian quadrature formula for this function $f$ is a rational function of $z$ and is exactly the $n$-th approximant ( $n$-th convergent) of the $J$-fraction for this Stieltjes transform and the convergence of the Gaussian quadrature formula for $f$ therefore gives an important result of Markov regarding the convergence of the diagonal in the Padé table for the Stieltjes transform of a positive measure.

Another important application of the Markov-Stieltjes inequalities is a necessary and sufficient condition for determinacy of the moment problem: if

$$
\sum_{n=0}^{\infty} p_{n}^{2}(x)=\infty
$$

for every real $x$ which is not a point of discontinuity of $\mu$, then the moment problem for $\mu$ is determinate. The Markov-Stieltjes inequalities are also very useful for estimations of the rate of convergence of the Gaussian quadrature formula; the Possé-Markov-Stieltjes inequalities even give results for singular integrands (Lubinsky and Rabinowitz [59]). The estimation of the distance between two succesive zeros of orthogonal (and also quasi-orthogonal) polynomials can also be done using these inequalities. From (4.3) one finds

$$
\lambda_{j, n}<\mu\left(x_{j+1, n}, x_{j-1, n}\right),
$$

which allowed Stieltjes to deduce that $\lambda_{j, n}$ tends to zero when $n \rightarrow \infty$ whenever the behaviour of $x_{j+1, n}-x_{j-1, n}$ is known in terms of the measure $\mu$. Stieltjes gave the result for Legendre polynomials. Nevai [68, p. 21] used the bound (4.5) to show that for measures $\mu$ with compact support such that for every $\epsilon>0$ the set $\operatorname{supp}(\mu) \cap(x-\epsilon, x+\epsilon)$ is an infinite set, there exists a sequence of integers $k_{n}(n=1,2, \ldots)$ such that

$$
\lim _{n \rightarrow \infty} x_{k_{n}, n}=x, \quad \lim _{n \rightarrow \infty} \lambda_{k_{n}, n}=0 .
$$

This shows that the zeros are dense in the derived set of $\operatorname{supp}(\mu)$ and that the corresponding Christoffel numbers tend to zero. For the isolated points in $\operatorname{supp}(\mu)$ Nevai $[68$, p. 156] used (4.3) to show that

$$
\lim _{\epsilon \rightarrow 0+} \limsup _{n \rightarrow \infty} \sum_{\left|x-x_{k, n}\right|<\epsilon} \lambda_{k, n}=\mu(\{x\}),
$$

for every $x \in \mathbb{R}$. Freud $[28$, p. 111] shows that for two consecutive zeros in an interval $[c, d]$ for which

$$
0<m<\frac{\mu(x, y]}{y-x} \leq M, \quad x, y \in[c, d]
$$


one has

$$
\frac{c_{1}}{n} \leq x_{j+1, n}-x_{j, n} \leq \frac{c_{2}}{n}
$$

where $c_{1}, c_{2}$ are positive constants. This is a slight extension of a result by Erdős and Turán [24]. Nevai [68, p. 164] generalizes this result by allowing $\mu^{\prime}$ to have an algebraic singularity inside $\operatorname{supp}(\mu)$. If $\operatorname{supp}(\mu)$ is compact, $\Delta \subset$ $\operatorname{supp}(\mu), t \in \Delta^{o}$ (the interior of the set $\Delta$ ) and if $\mu$ is absolutely continuous in $\Delta$ with

$$
c_{1}|x-t|^{\gamma} \leq \mu^{\prime}(x) \leq c_{2}|x-t|^{\gamma}, \quad \gamma>-1
$$

then

$$
\frac{c_{3}}{n} \leq x_{k, n}-x_{k-1, n} \leq \frac{c_{4}}{n}
$$

whenever $x_{k, n} \in \Delta_{1}$ with $\Delta^{1}$ a closed subset of $\Delta^{o}$. The Markov-Stieltjes inequalities are crucial to prove all these results.

\section{Special Polynomials}

\subsection{Legendre Polynomials}

Stieltjes wrote a number of papers directly related to the Legendre polynomials $P_{n}(x)$ for which

$$
\int_{-1}^{1} P_{n}(x) P_{m}(x) d x=0, \quad m \neq n .
$$

He always uses the notation $X_{n}$ but here we will adopt the notation $P_{n}$ which is nowadays standard. In [100] he uses the electrostatic interpretation of the zeros of Jacobi polynomials to obtain monotonicity properties of the zeros of Jacobi polynomials as a function of the parameters, and from this one easily finds bounds for the zeros $x_{1, n}>x_{2, n}>\cdots>x_{n, n}$ of the Legendre polynomials $P_{n}(x)=P_{n}^{(0,0)}(x)$ in terms of the zeros of the Jacobi polynomials $P_{n}^{\left(\frac{1}{2},-\frac{1}{2}\right)}(x)$ and $P_{n}^{\left(-\frac{1}{2}, \frac{1}{2}\right)}(x)$ giving

$$
\cos \frac{2 k \pi}{2 n+1}<x_{k, n}<\cos \frac{(2 k-1) \pi}{2 n+1}, \quad 1 \leq k \leq n .
$$

These bounds were already given by Bruns [11] in 1881 and Stieltjes does refer to Bruns' result, but Stieltjes goes on and shows that by using the zeros of Chebyshev polynomials of the first kind $T_{n}(x)=P_{n}^{\left(-\frac{1}{2},-\frac{1}{2}\right)}(x)$ and of the second kind $U_{n}(x)=P_{n}^{\left(\frac{1}{2}, \frac{1}{2}\right)}(x)$ one may find better bounds:

$$
\cos \frac{k \pi}{n+1}<x_{k, n}<\cos \frac{(2 k-1) \pi}{2 n}, \quad 1 \leq k \leq n / 2 .
$$

A great deal of work has been done to obtain sharp bounds for zeros of orthogonal polynomials. The monotonicity of zeros of orthogonal polynomials depending on a parameter is often used. Markov [61] [110, Theorem 6.12.1] 
gave a very nice result concerning the dependence of the zeros on a parameter $t$ which appears in the weight function $w(x)=w(x ; t)$. Two other methods for obtaining bounds for zeros of orthogonal polynomials are the Sturm comparison theorem [110, §6.3] [57] for solutions of Sturm-Liouville differential equations and the Hellman-Feynman theorem [45] of quantum chemistry. See also [44] for results on the monotonicity of zeros of orthogonal polynomials.

Stieltjes made a very important contribution concerning the asymptotic behaviour of Legendre polynomials. In 1878 Darboux [23] gave an asymptotic series for the Legendre polynomial:

$$
\begin{aligned}
& P_{n}(\cos \theta)=2 a_{n} \sum_{k=0}^{m-1} a_{k} \frac{1 \cdot 3 \cdots(2 k-1)}{(2 n-1)(2 n-3) \cdots(2 n-2 k+1)} \\
& \quad \times \frac{\cos \left[\left(n-k+\frac{1}{2}\right) \theta-\left(k+\frac{1}{2}\right) \pi / 2\right]}{(2 \sin \theta)^{k+\frac{1}{2}}}+O\left(n^{-m-\frac{1}{2}}\right), \quad 0<\theta<\pi,
\end{aligned}
$$

which generalizes an asymptotic formula given by Laplace (when $m=1$ ). Here

$$
a_{0}=1, \quad a_{k}=\frac{1 \cdot 3 \cdots(2 k-1)}{2^{k} k !} .
$$

The problem with this formula is that there is no closed expression or a bound on the error term. Moreover the infinite series actually converges in the ordinary sense when $\frac{\pi}{6}<\theta<\frac{5 \pi}{6}$, but it converges to $2 P_{n}(\cos \theta)$ rather than $P_{n}(\cos \theta)$ (this "paradox" was first pointed out by Olver [72]). This is probably the easiest example showing that asymptotic expansions need not converge to the function that they approximate. The reason why things go wrong here is that the formula is obtained by the so-called method of Darboux which consists of obtaining asymptotic results of a sequence by carefully examining the singularities on the circle of convergence of the generating function. The generating function of Legendre polynomials has two singularities on the circle of convergence, and at each singularity one picks up information on $P_{n}(\cos \theta)$. This is probably the reason why the convergence of the infinite series is to $2 P_{n}(\cos \theta)$ rather than $P_{n}(\cos \theta)$. Stieltjes' generalization of Laplace's asymptotic formula for the Legendre polynomials does not suffer from either problem. Stieltjes' asymptotic expansion is [102] [103]

$$
\begin{gathered}
P_{n}(\cos \theta)=\frac{4}{\pi} \frac{2^{n} n !}{3 \cdot 5 \cdots(2 n+1)} \\
\times \sum_{k=0}^{m-1} b_{k} \frac{\cos \left[\left(n+k+\frac{1}{2}\right) \theta-\left(k+\frac{1}{2}\right) \pi / 2\right]}{(2 \sin \theta)^{k+\frac{1}{2}}}+R_{m}(\theta), \quad 0<\theta<\pi,
\end{gathered}
$$

where

$$
b_{0}=1, \quad b_{k}=\frac{1^{2} \cdot 3^{2} \cdots(2 k-1)^{2}}{2^{k} k !(2 n+3)(2 n+5) \cdots(2 n+2 k+1)},
$$

and the error $R_{m}(\theta)$ is bounded by 


$$
\left|R_{m}(\theta)\right|<b_{m} \frac{4}{\pi} \frac{2^{n} n !}{3 \cdot 5 \cdots(2 n+1)} \frac{M}{(2 \sin \theta)^{m+\frac{1}{2}}},
$$

where

$$
M= \begin{cases}1 / \cos \theta, & \text { if } \sin ^{2} \theta \leq \frac{1}{2}, \\ 2 \sin \theta, & \text { if } \sin ^{2} \theta \geq \frac{1}{2}\end{cases}
$$

This asymptotic expansion converges in the ordinary sense when $\frac{\pi}{6}<\theta<\frac{5 \pi}{6}$ and it converges to $P_{n}(\cos \theta)$. Combined with Mehler's asymptotic formula

$$
\lim _{n \rightarrow \infty} P_{n}\left(\cos \frac{\theta}{n}\right)=J_{0}(\theta),
$$

one then finds an asymptotic series for the Bessel function $J_{0}$ already obtained by Poisson, but now with a bound on the error. Stieltjes also uses the asymptotic series to obtain approximations of the zeros of the Legendre polynomials. The asymptotic theory of orthogonal polynomials (in particular classical orthogonal polynomials) is very well developped nowadays, at least for orthogonality on a finite interval. Szegö has a very nice chapter on the asymptotic properties of the classical polynomials [110, Chapter VIII] and that book is still a very good source for asymptotic formulas for Jacobi, Laguerre and Hermite polynomials.

A third contribution of Stieltjes involving Legendre polynomials is his work on Legendre functions of the second kind [104]. The Legendre function of the second kind can be defined by

$$
Q_{n}(x)=\frac{1}{2} \int_{-1}^{1} \frac{P_{n}(y)}{x-y} d y, \quad x \in \mathbb{C} \backslash[-1,1],
$$

so that

$$
Q_{n}(x)=\frac{1}{2} P_{n}(x) \log \left(\frac{x+1}{x-1}\right)-P_{n-1}^{(1)}(x),
$$

where $P_{n-1}^{(1)}(x)$ is the associated Legendre polynomial of degree $n-1$. The integral representation cannot be used to define $Q_{n}(x)$ for $x \in[-1,1]$ but by taking the appropriate limit, or the appropriate branch of the logarithm, one can use (5.2) to define $Q_{n}(x)$ for $-1<x<1$. Hermite [41] had studied the zeros of $Q_{n}$ on $[-1,1]$ by making some changes of variables. Stieltjes works directly with $Q_{n}(x)$ as a function of the real variable $x$ and shows that $Q_{n}(x)$ has $n+1$ zeros in $(-1,1)$ which interlace with the zeros of the Legendre polynomial $P_{n}(x)$. He also shows that there can be no zeros outside $[-1,1]$ by using a simple property of Stieltjes transforms of positive weight functions. Some of these results can easily be generalized to functions of the second kind corresponding to general orthogonal polynomials [113].

\subsection{Stieltjes Polynomials}

In his last letter to Hermite [8, vol. II, pp. 439-441] Stieltjes considers the Legendre functions of the second kind (5.1) and observes that 


$$
\frac{1}{Q_{n}(z)}=E_{n+1}(z)+\frac{a_{1}}{z}+\frac{a_{2}}{z^{2}}+\cdots
$$

where $E_{n+1}(z)$ is a polynomial of degree $n+1$. This polynomial is now known as the Stieltjes polynomial ${ }^{2}$. Stieltjes gives the remarkable property

$$
\int_{-1}^{1} P_{n}(x) E_{n+1}(x) x^{k} d x=0, \quad 0 \leq k \leq n,
$$

which essentially means that $E_{n+1}(x)$ is orthogonal to all polynomials of degree less than or equal to $n$ with respect to the oscillating weight function $P_{n}(x)$ on $[-1,1]$. One may now wonder which properties of ordinary orthogonal polynomials are still valid for $E_{n+1}(x)$ and Stieltjes conjectures that the zeros of $E_{n+1}(x)$ are real, simple and belong to $[-1,1]$ and that they interlace with the zeros of $P_{n}(x)$. These conjectures were later proved by Szegö [109]. Szegö also extended the idea to ultraspherical weights by considering the functions of the second kind

$$
q_{n}^{\mu}(z)=\frac{1}{2} \frac{\Gamma(2 \mu)}{\Gamma\left(\mu+\frac{1}{2}\right)} \int_{-1}^{1}\left(1-t^{2}\right)^{\mu-\frac{1}{2}} \frac{P_{n}^{\mu}(x)}{z-x} d x
$$

where $P_{n}^{\mu}(x)$ is an ultraspherical polynomial of degree $n$. One can then find

$$
\frac{1}{q_{n}^{\mu}(z)}=E_{n+1}^{\mu}(z)+\frac{a_{1}^{\mu}}{z}+\frac{a_{2}^{\mu}}{z^{2}}+\cdots
$$

where $E_{n+1}^{\mu}(z)$ is a polynomial of degree $n+1$. Szegó shows that

$$
\int_{-1}^{1}\left(1-x^{2}\right)^{\mu-\frac{1}{2}} P_{n}^{\mu}(x) E_{n+1}^{\mu}(x) x^{k} d x=0, \quad 0 \leq k \leq n,
$$

thus generalizing the orthogonality of Stieltjes polynomials. The properties of the zeros of $E_{n+1}^{\mu}(x)$ depend on the value of the parameter $\mu$. If $0<\mu \leq 2$ then the zeros of $E_{n+1}^{\mu}(x)$ are in $[-1,1]$, they are real and simple and they interlace with the zeros of $P_{n}^{\mu}(x)$. When $\mu<0$ then some of the zeros are outside $[-1,1]$ and Monegato [66] has made some computations showing that for $\mu \geq 4.5$ there can be complex zeros, depending on the degree $n$. More precise numerical information for Gegenbauer weights as well as for Jacobi weights has been obtained by Gautschi and Notaris [32]. The construction of Stieltjes and Szegő can be generalized by considering a positive measure $\mu$ on $\mathbb{R}$. Suppose that $p_{n}(x ; \mu)(n=0,1,2, \ldots)$ are the orthogonal polynomials with respect to the measure $\mu$, then the functions of the second kind are

$$
q_{n}(z ; \mu)=\int \frac{p_{n}(x ; \mu)}{z-x} d \mu(x)
$$

$\overline{2}$ The polynomial solutions of a Lamé differential equation (3.5) are also known as Stieltjes polynomials but we will not use that terminology. 
and these are defined for $z \in \mathbb{C} \backslash \operatorname{supp}(\mu)$. Define the (general) Stieltjes polynomial $E_{n+1}(z ; \mu)$ by

$$
\frac{1}{q_{n}(z ; \mu)}=E_{n+1}(z ; \mu)+\frac{a_{1}(\mu)}{z}+\frac{a_{2}(\mu)}{z^{2}}+\cdots,
$$

then one always has

$$
\int_{-1}^{1} p_{n}(x ; \mu) E_{n+1}(x ; \mu) x^{k} d \mu(x)=0, \quad 0 \leq k \leq n .
$$

These Stieltjes polynomials turn out to have some importance in constructing an optimal pair $(A, B)$ of quadrature formulas. Suppose we start with a quadrature formula $A$ with $n$ nodes and a quadrature formula $B$ with $m$ nodes $(m>n)$. In order to compute the error of formula $A$ one often assumes that the difference of the results obtained by using $A$ and $B$ is proportional to the actual error of the quadrature formula $A$. This means that one needs $n+m$ function evaluations to compute the error of $A$. This implies that one has done $m$ extra function evaluations which are not used in the evaluation of $A$ itself. Kronrod [55] suggested to extend formula $B$ to a formula with $n+m$ nodes in such a way that the accuracy of $B$ is as high as possible. For the Legendre weight on $[-1,1]$ one will find an optimal pair $(A, B)$ by taking for $A$ the Gaussian quadrature with nodes equal to the zeros of the Legendre polynomial $P_{n}(x)$ and for $B$ a quadrature formula with $2 n+1$ nodes at the zeros of $P_{n}(x)$ and the zeros of $E_{n+1}(x)$. The quadrature formula $B$ then turns out to give a correct result for all polynomials of degree less than or equal to $3 n+1$ [55, Theorem 6].

In 1930 Geronimus [33] slightly changes Stieltjes' idea and considers the Jacobi functions of the second kind

$$
Q_{n}^{(\alpha, \beta)}(z)=\int_{-1}^{1} \frac{P_{n}^{(\alpha, \beta)}(x)}{z-x}(1-x)^{\alpha}(1+x)^{\beta} d x,
$$

with $P_{n}^{(\alpha, \beta)}(x)$ the Jacobi polynomial of degree $n$. Geronimus observes that

$$
\frac{1}{Q_{n}(z) \sqrt{z^{2}-1}}=S_{n}(z)+\frac{c_{1}}{z}+\frac{c_{2}}{z}+\cdots
$$

with $S_{n}(z)$ a polynomial of degree $n$. Notice the extra factor $\sqrt{z^{2}-1}$ in the denominator on the left hand side. These polynomials satisfy the remarkable property

$$
\int_{-1}^{1}(1-x)^{\alpha}(1+x)^{\beta} P_{n}^{(\alpha, \beta)}(x) S_{n}(x) T_{k}(x) d x=0, \quad 0<k \leq n,
$$

and

$$
\int_{-1}^{1}(1-x)^{\alpha}(1+x)^{\beta} P_{n}^{(\alpha, \beta)}(x) S_{n}(x) d x=1 .
$$


Here $T_{k}(x)$ is the Chebyshev polynomial of the first kind of degree $k$. Geronimus polynomials can be generalized to other weights on $[-1,1]$. The interval $[-1,1]$ is important because it accounts for the factor $\sqrt{z^{2}-1}$ in the definition of the Geronimus polynomials. There is a relation between the Geronimus polynomials $S_{n}(x)$ and the Stieltjes polynomials $E_{n+1}(x)$ if one works with a weight function on $[-1,1]$ : if

$$
E_{n+1}(x)=\sum_{k=0}^{n+1} c_{k, n} T_{k}(x),
$$

(the prime means to divide the first term by two) is the expansion of $E_{n+1}(x)$ in Chebyshev polynomials of the first kind, then

$$
S_{n}(x)=\sum_{k=0}^{n} c_{k+1, n} U_{k}(x)
$$

is the expansion of $S_{n}(x)$ in Chebyshev polynomials of the second kind.

Stieltjes and Geronimus polynomials and the related Gauss-Kronrod quadrature are still being studied and we refer to Gautschi [31], Monegato [66], Peherstorfer [76] [77] and Prévost [82] for more information.

\subsection{Stieltjes-Wigert Polynomials}

In his memoir $[105, \S 56]$ Stieltjes explicitly gives an example of a moment problem on $[0, \infty)$ which is indeterminate. He shows that

$$
\int_{0}^{\infty} u^{k} u^{-\log u}[1+\lambda \sin (2 \pi \log u)] d u=\sqrt{\pi} e^{\frac{(k+1)^{2}}{4}}
$$

is independent of $\lambda$ and therefore the weight functions

$$
w_{\lambda}(u)=u^{-\log u}[1+\lambda \sin (2 \pi \log u)], \quad-1 \leq \lambda \leq 1
$$

all have the same moments which implies that this moment problem is indeterminate. Stieltjes gives the coefficients of the continued fraction (1.1)

$$
c_{2 n}=(q ; q)_{n-1} q^{n}, \quad c_{2 n+1}=\frac{q^{\frac{2 n+1}{2}}}{(q ; q)_{n}},
$$

where $q=e^{-1 / 2}$ and

$$
(a ; q)_{0}=1, \quad(a ; q)_{n}=(1-a)(1-a q)\left(1-a q^{2}\right) \cdots\left(1-a q^{n-1}\right) .
$$

Both the series $\sum c_{2 n}$ and $\sum c_{2 n+1}$ converge since $0<q<1$, which agrees with the theory worked out by Stieltjes. Later Wigert [122] extended this by considering the weight functions

$$
w_{k}(x)=e^{-k^{2} \log ^{2} x}, \quad 0<x<\infty,
$$


which for $k=1$ reduce to the weight function considered by Stieltjes. If we set $q=e^{-1 /\left(2 k^{2}\right)}$ then the orthogonal polynomials are given by

$$
p_{n}(x)=\sum_{j=0}^{n} \frac{\left(q^{-n} ; q\right)_{j}}{(q ; q)_{j}} q^{j^{2} / 2}\left(q^{n+1} x\right)^{j},
$$

and are known as Stieltjes-Wigert polynomials. The moment problem is indeterminate whenever $0<q<1$, which means that there exist an infinite number of measures on $[0, \infty)$ with the same moments. Askey [7] indicated that these polynomials are related to theta functions and shows that the weight function

$$
w(x)=\frac{x^{-5 / 2}}{(-x ; q)_{\infty}(-q / x ; q)_{\infty}}, \quad 0<x<\infty
$$

has the same moments. This measure arises as a $q$-extension of the beta density on $[0, \infty)$. Chihara [16] [18] has given many more measures which have the same moments as the weight function $w_{k}(x)$ given by Wigert.

The Stieltjes-Wigert polynomial $p_{n}(x)$ is a (terminating) basic hypergeometric series. Such series are of the form $\sum c_{j}$ with $c_{j+1} / c_{j}$ a rational function of $q^{j}$ for a fixed $q$ (for hypergeometric series this ratio is a rational function of $j$ ). The first set of orthogonal polynomials which are basic hypergeometric series was found by Markov in his thesis [63]. Except for a reference in Szegö's book $[110, \S 2.9]$, this work was overlooked and seems not to have led to any extensions. Markov's polynomials are discrete extensions of Legendre polynomials and basic hypergeometric extensions of discrete Chebyshev polynomials which are orthogonal on $\{0,1,2, \ldots, N\}$ with respect to the uniform distribution. They are a special case of polynomials considered by Hahn, which will be mentioned later. The next basic hypergeometric orthogonal polynomials were introduced in 1894, and there were two different examples that year. These are the Stieltjes-Wigert polynomials (with $q=e^{-1 / 2}$ ) given by Stieltjes and the continuous $q$-Hermite polynomials given by Rogers [87]. Both are basic hypergeometric extensions of Hermite polynomials but of a completely different nature. Those of Rogers are orthogonal on $[-1,1]$ with respect to the weight function

$$
w(x)=\frac{\prod_{k=0}^{\infty}\left[1-2\left(2 x^{2}-1\right) q^{k}+q^{2 k}\right]}{\sqrt{1-x^{2}}} .
$$

A number of other examples were found before Hahn [36] considered the following problem: find all sets of orthogonal polynomials $p_{n}(x)(n=0,1,2, \ldots)$ such that

$$
r_{n}(x)=\frac{p_{n+1}(x)-p_{n+1}(q x)}{x}, \quad n=0,1,2, \ldots
$$

is again a set of orthogonal polynomials. Earlier it had been shown that if $p_{n}(x)$ $(n=0,1,2, \ldots)$ are orthogonal and $p_{n+1}^{\prime}(x)(n=0,1,2, \ldots)$ are orthogonal, then $p_{n}(x)(n=0,1,2, \ldots)$ are either Jacobi, Laguerre or Hermite polynomials (after a possible change of scale). It is easy to see that the Stieltjes-Wigert polynomials are in the Hahn class. The continuous $q$-Hermite polynomials 
of Rogers are not in the Hahn class, but their analogous difference operator is a divided difference operator. Basic hypergeometric series and orthogonal polynomials which are terminating basic hypergeometric series are described in detail in the book by Gasper and Rahman [29]. All of these polynomials arise in the study of quantum groups (see Koornwinder [52] and references there).

\subsection{Orthogonal Polynomials Related to Elliptic Functions}

In Chapter XI of his memoir [105] Stieltjes gives some examples of continued fractions and the corresponding moment problem. These examples (except for one) had already been worked out in one of his previous papers [101]. The continued fractions are for the functions

$$
\begin{gathered}
F_{1}(z, k)=\int_{0}^{\infty} \operatorname{cn}(u, k) e^{-z u} d u, \quad F_{2}(z, k)=\int_{0}^{\infty} \operatorname{dn}(u, k) e^{-z u} d u, \\
F_{3}(z, k)=\int_{0}^{\infty} \operatorname{sn}(u, k) e^{-z u} d u, \quad F_{4}(z, k)=z \int_{0}^{\infty} \operatorname{sn}^{2}(u, k) e^{-z u} d u,
\end{gathered}
$$

which are all Laplace transforms of the Jacobian elliptic functions given by

$$
\begin{gathered}
\operatorname{cn}(u, k)=\cos \varphi=\frac{2 \pi}{k K} \sum_{n=1}^{\infty} \frac{q^{n-\frac{1}{2}}}{1+q^{2 n-1}} \cos \frac{(2 n-1) \pi u}{2 K}, \\
\operatorname{sn}(u, k)=\sin \varphi=\frac{2 \pi}{k K} \sum_{n=1}^{\infty} \frac{q^{n-\frac{1}{2}}}{1-q^{2 n-1}} \sin \frac{(2 n-1) \pi u}{2 K}, \\
\operatorname{dn}(u, k)=\sqrt{1-k^{2} \sin ^{2} \varphi}=\frac{\pi}{2 K}+\frac{2 \pi}{K} \sum_{n=1}^{\infty} \frac{q^{n}}{1+q^{2 n}} \cos \frac{n \pi u}{K},
\end{gathered}
$$

with

$$
u=\int_{0}^{\varphi} \frac{d \theta}{\sqrt{1-k^{2} \sin ^{2} \theta}}
$$

and

$$
q=e^{-\pi K^{\prime} / K}, \quad K(k)=\int_{0}^{1} \frac{d x}{\sqrt{\left(1-x^{2}\right)\left(1-k^{2} x^{2}\right)}}, \quad K^{\prime}(k)=K\left(1-k^{2}\right) .
$$

The Chudnovsky's [20, p. 197] pointed out that these continued fractions are some of the very rare cases where both the function and its continued fraction expansion are known explicitly. There are quite a few cases known when the function is given in terms of (basic) hypergeometric series and the numerators and denominators of the convergents of the continued fraction are classical orthogonal polynomials (in Askey's definition). The three-term recurrence relation then gives the coefficients of the $J$-fraction. The functions $F_{i}(z, k)(i=1,2,3,4)$ however are not of (basic) hypergeometric type and the corresponding orthogonal polynomials are therefore not classical. Nevertheless 
Stieltjes succeeded in finding the continued fractions: he obtained $S$-fractions for $F_{1}$ and $F_{2}$ and $J$-fractions for $F_{3}$ and $F_{4}$. His method consists of decomposing a quadratic form with infinitely many variables as a sum of squares:

$$
\begin{aligned}
\sum_{i=0}^{\infty} \sum_{j=0}^{\infty} a_{i+j} x_{i} x_{j}= & c_{0}\left(x_{0}+a_{0,1} x_{1}+a_{0,2} x_{2}+\cdots\right)^{2} \\
& +c_{1}\left(x_{1}+a_{1,2} x_{2}+a_{1,3} x_{3}+\cdots\right)^{2} \\
& +c_{2}\left(x_{2}+a_{2,3} x_{3}+a_{2,4} x_{4}+\cdots\right)^{2}+\cdots
\end{aligned}
$$

The coefficients of the $J$-fraction of

$$
\sum_{n=0}^{\infty} \frac{(-1)^{n} a_{n}}{z^{n+1}}
$$

are then determined by the coefficients $c_{0}, c_{1}, \ldots$ and $a_{i, i+1}(i=0,1,2, \ldots)$. Such a decomposition can easily be made when the function

$$
f(x)=\sum_{n=0}^{\infty} a_{n} \frac{x^{n}}{n !}
$$

satisfies an addition formula of the type

$$
f(x+y)=c_{0} f(x) f(y)+c_{1} f_{1}(x) f_{1}(x)+c_{2} f_{2}(x) f_{2}(y)+\cdots
$$

where $f_{m}(x)=O\left(x^{m}\right)$, as Rogers [88] pointed out when he was reviewing Stieltjes' technique. The addition formulas for the Jacobian elliptic functions then readily give the desired continued fractions. The orthogonal polynomials that appear are defined by the recurrence relations

$$
C_{n+1}(x)=x C_{n}(x)-\alpha_{n} C_{n-1}(x), \quad D_{n+1}(x)=x D_{n}(x)-\beta_{n} D_{n-1}(x),
$$

with

$$
\alpha_{2 n}=(2 n)^{2} k^{2}, \quad \alpha_{2 n+1}=(2 n+1)^{2}, \quad \beta_{2 n}=(2 n)^{2}, \quad \beta_{2 n+1}=(2 n+1)^{2} k^{2} .
$$

These polynomials have later been studied in detail by Carlitz [14]. The generating function for the orthogonal polynomials satisfies a Lamé differential equation

$$
y^{\prime \prime}+\frac{1}{2}\left\{\frac{1}{x}+\frac{1}{x-1}+\frac{1}{x-a}\right\} y^{\prime}+\frac{b-n(n+1) x}{4 x(x-1)(x-a)} y=0,
$$

with $n=0$ (in general $n$ is an integer). There exist $2 n+1$ values of the parameter $b$ for which this Lamé equation has algebraic function solutions. Stieltjes approach has been generalized to continued fraction expansions for which the generating functions of the numerators and denominators of the convergents satisfy a Lamé differential equation with $n \neq 0$. This has been done by the Chudnovsky brothers [20, pp. 197-201] [21, §13]. Some of these generalizations have interesting applications in number theory: the irrationality and bounds on the measure of irrationality of some values of complete elliptic integrals of the third kind can be obtained from these continued fraction expansions. 


\section{References}

1. Euvres Complètes de Thomas Jan Stieltjes, vol. I, P. Noordhoff, Groningen, 1914

2. Euvres Complètes de Thomas Jan Stieltjes, vol. II, P. Noordhoff, Groningen, 1918

3. Akhiezer, N. I.: The Classical Moment Problem, Oliver and Boyd, Edinburgh, 1965

4. Alam, M.: Zeros of Stieltjes and Van Vleck polynomials, Trans. Amer. Math. Soc. 252 (1979), 197-204

5. Al-Rashed, A. M. and Zaheer, N.: Zeros of Stieltjes and Van Vleck polynomials and applications, J. Math. Anal. Appl. 110 (1985), 327-339

6. Aomoto, K.: Jacobi polynomials associated with Selberg integrals, SIAM J. Math. Anal. 18 (1987), 545-549

7. Askey, R.: Orthogonal polynomials and theta functions, in "Theta Functions, Bowdoin 1987" (part 2) (Ehrenpreis, L. and Gunning, R. C., eds.) Proceedings of Symposia in Pure Mathematics 49, Amer. Math. Soc., Providence, RI, 1989, pp. 299-321

8. Baillaud, B. and Bourget, H.: Correspondance d'Hermite et de Stieltjes, vol. I-II, Gauthier-Villars, Paris, 1905

9. Berg, C. and Thill, M.: Rotation invariant moment problems, Acta Math. 167 (1991), 207-227

10. Brezinski, C.: History of Continued Fractions and Padé Approximants, Springer Series in Computational Mathematics 12, Springer-Verlag, Berlin, 1991

11. Bruns, H.: Zur Theorie der Kugelfunktionen, J. Reine Angew. Math. 90 (1881), $322-328$

12. Carleman, T.: Sur le problème des moments, C.R. Acad. Sci. Paris 174 (1922), $1680-1682$

13. _ Sur les équations intégrales singulières à noyau réel et symmétrique, Uppsala Universitets Årsskrift, 1923, 228 pp.

14. Carlitz, L.: Some orthogonal polynomials related to elliptic functions, Duke Math. J. 27 (1960), 443-460

15. Chebyshev, P. L.: Sur les valeurs limites des intégrales, J. Math. (2) 19 (1874), $157-160$

16. Chihara, T. S.: A characterization and a class of distribution functions for the Stieltjes-Wigert polynomials, Canad. Math. Bull. 13 (1970), 529-532

17. $\quad$ : An Introduction to Orthogonal Polynomials, Gordon and Breach, New York, 1978

18. _ : On generalized Stieltjes-Wigert and related orthogonal polynomials, J. Comput. Appl. Math. 5 (1979), 291-297

19. Christoffel, E. B.: Sur une classe particulière de fonctions entières et de fonctions continues, Ann. Mat. Pura Appl. (2) 8 (1877), 1-10 
20. Chudnovsky, D. V. and Chudnovsky G. V.: Transcendental methods and thetafunctions, in "Theta Functions, Bowdoin 1987" (part 2) (Ehrenpreis, L. and Gunning, R.C., eds.), Proceedings of Symposia in Pure Mathematics 49, Amer. Math. Soc., Providence, RI, 1989, pp. 167-232

21. _ C Computer assisted number theory with applications, Lecture Notes in Mathematics 1240, Springer-Verlag, Berlin, 1987, pp. 1-68

22. Cosserat, E.: Notice sur les travaux scientifiques de Thomas-Jean Stieltjes, Ann. Fac. Sci. Tolouse 9 (1895), 1-64

23. Darboux, G.: Mémoire sur l'approximation des fonctions de très grands nombres, J. Math. (3) 4 (1878), 5-56; 377-416

24. Erdős, P. and Turán, P.: On interpolation II, Ann. of Math. 39 (1938), 703-724

25. Favard, J.: Sur les polynômes de Tchebicheff, C.R. Acad. Sci. Paris 200 (1935), 2052-2053

26. Fekete, M.: Über die Verteilung der Wurzeln bei gewissen algebraischen Gleichungen mit ganzzahligen Koefficienten, Math. Z. 17 (1923), 228-249

27. Forrester, P. J. and Rogers J. B.: Electrostatics and the zeros of the classical polynomials, SIAM J. Math. Anal. 17 (1986), 461-468

28. Freud, G.: Orthogonal Polynomials, Akadémiai Kiadó and Pergamon Press, Budapest and Oxford, 1971

29. Gasper, G. and Rahman, M.: Basic Hypergeometric Series, Encyclopedia of Mathematics and its Applications 35, Cambridge University Press, Cambridge, 1990

30. Gautschi, W.: A survey of Gauss-Christoffel quadrature formulae, in "E.B. Christoffel: the Influence of his Work on Mathematics and the Physical Sciences" (P. L. Butzer, F. Fehér, eds.), Birkhäuser, Basel, 1981, pp. 72-147

31. _ G Gauss-Kronrod quadrature - a survey, in "Numerical Methods and Approximation Theory III" (G. V. Milovanović, ed.), Faculty of Electronic Engineering, University of Niš, 1988, pp. 39-66

32. Gautschi, W. and Notaris S. E.: An algebraic study of Gauss-Kronrod quadrature formulae for Jacobi weight functions, Math. Comp. 51 (1988), 231-248

33. Geronimus, Ya. L.: On a set of polynomials, Ann. of Math. 31 (1930), 681-686

34. Gonchar, A. A. and Rakhmanov, E. A.: Equilibrium measure and the distribution of zeros of extremal polynomials, Math. USSR Sb. 53 (1986), 119-130

35. Gustafson, R. A.: A generalization of Selberg's beta integral, Bull. Amer. Math. Soc. 22 (1990), 97-105

36. Hahn, W.: Über Orthogonalpolynome die $q$-Differenzengleichungen genügen, Math. Nachr. 2 (1949), 4-34

37. Hamburger, H.: Über eine Erweiterung des Stieltjesschen Momentenproblems, Math. Ann. 81 (1920), 235-318; 82 (1921), 120-164; 168-187

38. Hardy, G. H.: Notes on some points in the integral calculus, XLVI On Stieltjes 'problème des moments', Messenger of Math. 46 (1917), 175-182; XLVII On Stieltjes 'problème des moments' (cont.), Messenger of Math. 47 (1918), 81-88

39. Hausdorff, F.: Momentprobleme für ein endliches Intervall, Math. Z. 16 (1923), $220-248$

40. Hendriksen, E. and van Rossum, H.: Electrostatic interpretation of zeros, in "Orthogonal Polynomials and their Applications", Lecture Notes in Mathematics 1329, (Alfaro et al., eds.), Springer-Verlag, Berlin, 1988, pp. 241-250

41. Hermite, Ch.: Sur les racines de la fonction sphérique de seconde espèce, Ann. Fac. Sci. Toulouse 4 (1890), I1-10

42. Hilbert, D.: Über die Discriminante der im Endlichen abbrechenden hypergeometrische Reihen, J. Reine Angew. Math. 103 (1888), 337-345

43. __ Grundzüge einer allgemeinen Theorie der linearen Integralgleichungen, Leipzig, 1912; Chelsea, New York, 1953 
44. Ismail M. E. H. and Muldoon M. E.: A discrete approach to monotonicity of zeros of orthogonal polynomials, Trans. Amer. Math. Soc. 323 (1991), 65-78

45. Ismail, M. E. H. and Zhang, R.: On the Hellman-Feynman theorem and the variation of zeros of certain special functions, Adv. Appl. Math. 9 (1988), 439446

46. Jones W. B. and Thron, W. J.: Continued Fractions: Analytic Theory and Applications, Encyclopedia of Mathematics and its applications 11, AddisonWesley, Reading, Massachusetts, 1980

47. Jones, W. B., Thron, W. J. and Njåstad, O.: Orthogonal Laurent polynomials and the strong Hamburger moment problem, J. Math. Anal. Appl. 98 (1984), $528-554$

48. Jones, W. B., Thron, W. J. and Waadeland, H.: A strong Stieltjes moment problem, Trans. Amer. Math. Soc. 261 (1980), 503-528

49. Karlin, S. and McGregor, J.: The differential equations of birth and death processes and the Stieltjes moment problem, Trans. Amer. Math. Soc. 85 (1957), $489-546$

50. Karlin, S. and Shapley, L. S.: Geometry of Moment Spaces, Memoirs Amer. Math. Soc. 12, Providence, R.I., 1953

51. Karlin, S. and Studden, W. J.: Tchebycheff Systems: with Applications in Analysis and Statistics, Interscience, New York, 1966

52. Koornwinder, T. H.: Orthogonal polynomials in connection with quantum groups, in "Orthogonal Polynomials: Theory and Parctice" [69], pp. 257-292

53. Krein, M. G.: The ideas of P. L. Chebysheff and A. A. Markov in the theory of limiting values of integrals and their further development, Amer. Math. Soc. Transl. Series 2, 12, Amer. Math. Soc., Providence, RI, 1959, pp. 1-122

54. Krein, M. G. and Nudelman, A. A.: The Markov Moment Problem and Extremal Properties, Translations of Mathematical Monographs 50, Amer. Math. Soc., Providence, RI, 1977

55. Kronrod, A. S.: Nodes and Weights for Quadrature Formulas, Nauka, Moscow, 1964 (Russian); Consultants Bureau, New York, 1965

56. Ledermann, W. and Reuter, G. E. H.: Spectral theory for the differential equations of simple birth and death processes, Philos. Trans. Roy. Soc. London Ser. A 246 (1954), 321-369

57. Lorch, L.: Elementary comparison techniques for certain classes of SturmLiouville equations, Differential equations, Sympos. Univ. Upsaliensis Ann. Quigentesimum Celebrantis 7, Almqvist and Wiksell, Stockholm, 1977, pp. $125-133$

58. Lorentzen, L. and Waadeland, H.: Continued Fractions and Some of its Applications, North-Holland Studies in Computational Mathematics, North-Holland, 1991 (to appear)

59. Lubinsky, D. S. and Rabinowitz, P.: Rates of convergence of Gaussian quadrature for singular integrands, Math. Comp. 43 (1984), 219-242

60. Macdonald, I. G.: Orthogonal polynomials associated with root systems, in "Orthogonal Polynomials: Theory and Practice" [69], pp. 311-318

61. Markov, A. A.: Sur les racines de certaines équations, Math. Ann. 27 (1886), $177-182$

62. _ Démonstration de certaines inégalités de M. Tchébycheff, Math. Ann. 24 (1884), 172-180

63. _ : On Some Applications of Algebraic Continued Fractions, Thesis (in Russian), St. Petersburg, 1884, 133 pp.

64. Mehta, M. L.: Random Matrices and the Statistical Theory of Energy Levels, Academic Press, New York, 1967 (2nd edition), 1990 (revised and enlarged 2nd edition)

65. Mhaskar, H. N. and Saff, E. B.: Where does the $L^{p}$-norm of a weighted polynomial live? Trans. Amer. Math. Soc. 303 (1987), 109-124 
66. Monegato, G.: Stieltjes polynomials and related quadrature rules, SIAM Review 24 (1982), 137-157

67. Montel, P.: Leçons Sur les Familles Normales de Fonctions Analytiques et leurs Applications, Gauthier-Villars, Paris, 1927

68. Nevai, P. G.: Orthogonal Polynomials, Memoirs Amer. Math. Soc. 213, Providence, RI, 1979

69. Nevai, P. (ed.): Orthogonal Polynomials: Theory and Practice, NATO-ASI series C 294, Kluwer, Dordrecht, 1990

70. Nevanlinna, R.: Asymptotische Entwickelungen beschränkter Funktionen und das Stieltjessche Momentenproblem, Ann. Acad. Sci. Fenn. Ser. A 18 (1922), no. 5 (52 pp.)

71. Njåstad, O.: An extended Hamburger moment problem, Proc. Edinburgh Math. Soc. 28 (1985), 167-183

72. Olver, F. W. J.: A paradox in asymptotics, SIAM J. Math. Anal. 1 (1970), 533-534

73. Padé, H.: Sur la représentation approchée d'une fonction par des fractions rationelles, Ann. Sci. École Norm. Sup. (3) 9 (1892) (supplément), 1-93

74. 911-912

75. Pastro, P. L.: Orthogonal polynomials and some $q$-beta integrals of Ramanujan, J. Math. Anal. Appl. 112 (1985), 517-540

76. Peherstorfer, F.: On Stieltjes polynomials and Gauss-Kronrod quadrature, Math. Comp. 55 (1990), 649-664

77. _ : On the asymptotic behaviour of functions of the second kind and Stieltjes polynomials and on Gauss-Kronrod quadrature formulas, J. Approx. Theory (to appear)

78. Perron, O.: Die Lehre von den Kettenbrüchen, Teubner, Leipzig, 1913 (band I, 1954; band II, 1957); Chelsea, New York, 1950

79. Pólya, G.: Sur un théorème de Stieltjes, C.R. Acad. Sci. Paris 155 (1912), 767-769

80. Possé, K.: Sur les quadratures, Nouvelles Ann. Math. (2) 14 (1875), 49-62

81. _ Sur Quelques Applications des Fractions Continues Algébriques, Hermann, Paris, 1886

82. Prévost, M.: Stieltjes type and Geronimus type polynomials, J. Comput. Appl. Math. 21 (1988), 133-144

83. Pringsheim, A.: Über die Konvergenz unendlicher Kettenbrüche, S.B. Bayer. Akad. Wiss. Math.-Nat. Kl. 28 (1898), 295-324

84. __ : Über einige Konvergenzkriterien für Kettenbrüche mit komplexen Gliedern, S.B. Bayer. Akad. Wiss. Math.-Nat. Kl. 35 (1905), 359-380

85. Riesz, M.: Sur le problème des moments, Ark. Mat. Ast. Fys. 16 (1921), no. 12 (23 pp.); 16 (1922), no. 19 (21 pp.); 17 (1923), no. 16 (52 pp.)

86. _ : Sur le problème des moments et le théorème de Parseval correspondant, Acta Litt. Acad. Sci. (Szeged) 1 (1922-1923), 209-225

87. Rogers, L. J.: Second memoir on the expansion of certain infinite products, Proc. London Math. Soc. 25 (1894), 318-343

88. _ On the representation of certain asymptotic series as convergent continued fractions, Proc. London Math. Soc. (2) 4 (1907), 72-89

89. Schur, I.: Über die Verteilung der Wurzeln bei gewissen algebraischen Gleichungen mit ganzzahligen Koefficienten, Math. Z. 1 (1918), 377-402

90. Selberg, A.: Bemerkinger om et multipelt integral, Norsk Mat. Tidsskr. 26 (1944), 71-78

91. Shohat, J. A., Hille, E. and Walsh, J. L.: A Bibliography on Orthogonal Polynomials, Bulletin of the National Research Council 103, National Research Council of the National Academy of Sciences, Washington, D.C., 1940 
92. Shohat, J. A. and Tamarkin, J. D.: The Problem of Moments, Amer. Math. Soc., Providence, RI, 1963

93. Siegel, C. L.: The trace of totally positive and real algebraic integers, Ann. of Math. 46 (1945), 302-312

94. Stahl, H. and Totik, V.: General Orthogonal Polynomials, Encyclopedia of Mathematics and its Applications, Cambridge University Press, 1992

95. Stieltjes, T.J.: Quelques recherches sur la théorie des quadratures dites mécaniques, Ann. Sci. École Norm. Sup. (3) 1 (1884), 409-426

96. _ : Note à l'occasion de la réclamation de M. Markoff, Ann. Sci. École Norm. Sup. (3) 2 (1885), 183-184

97. _ : Sur quelques théorèmes d'algèbre, C.R. Acad. Sci. Paris 100 (1885), $439-440$

98. _ : Sur les polynômes de Jacobi, C.R. Acad. Sci. Paris 100 (1885), 620622

99. _ S Sur certaines polynômes qui verifient une equation différentielle linéair du second ordre et sur la théorie des fonctions de Lamé, Acta Math. 6 (1885), $321-326$

100. _ Sur les racines de l'équation $X_{n}=0$, Acta Math. 9 (1886), 385-400

101. _ S Sur la réduction en fraction continue d'une série procédant suivant les puissances descendantes d'une variable, Ann. Fac. Sci. Toulouse 3 (1889), H1-17

102. _ Sur la valeur asymptotique des polynômes de Legendre, C.R. Acad. Sci. Paris 110 (1890), 1026-1027

103. G1-17

104. _ S Sur les racines de la fonction sphérique de seconde espèce, Ann. Fac. Sci. Toulouse 4 (1890), J1-10

105. (1894), J1-122; 9 (1895), A1-47

106. _ Sur certaines inégalités dues à M. P. Tchebychef, Euvres Complètes de Thomas Jan Stieltjes, vol. II [2], 586-593

107. Stone, M. H.: Linear Transformations in Hilbert Space and their Applications to Analysis, Amer. Math. Soc. Colloq. Publ. 15,

108. Szegö, G.: Bemerkungen zu einer Arbeit von Herrn M. Fekete: "Über die Verteilung der Wurzeln bei gewissen algebraischen Gleichungen mit ganzzahligen Koefficienten", Math. Z. 21 (1924), 203-208

109. _ _ Über gewisse orthogonale Polynome, die zu einer oszillierenden Belegungsfunktion gehören, Math. Ann. 110 (1934), 501-513

110. : Orthogonal Polynomials, Amer. Math. Soc. Colloq. Publ. 23, Amer. Math. Soc., Providence, RI, 1975 (4th edition)

111. Tsuji, M.: Potential Theory in Modern Function Theory, Chelsea, New York, 1959

112. Ullman, J. L.: On the regular behaviour of orthogonal polynomials, Proc. London Math. Soc. (3) 24 (1972), 119-148

113. Van Assche, W.: Orthogonal polynomials, associated polynomials and functions of the second kind, J. Comput. Appl. Math. 37 (1991), 237-249

114. Van Vleck, E. B.: On the polynomials of Stieltjes, Bull. Amer. Math. Soc. (2) 4 (1898), 426-438

115. _ : On the convergence of continued fractions with complex coefficients, Trans. Amer. Math. Soc. 2 (1901), 215-233

116. $\quad$ : On an extension of the 1894 memoir of Stieltjes, Trans. Amer. Math. Soc. 4 (1903), 297-332 
117. Vilenkin, N. Ya.: Special Functions and the Theory of Group Representations, Translations of Mathematical Monographs 22, Amer. Math. Soc., Providence, RI, 1968

118. Vitali, G.: Sopra le serie di funzioni analitiche, Rend. R. Ist. Lombardo 36 (1903), 771-774; Ann. Mat. Pura Appl. 10 (1904), 73

119. Wall, H. S.: Analytic Theory of Continued Fractions, Chelsea, Bronx, NY, 1973

120. Widom, H.: Polynomials associated with measures in the complex plane, J. Math. Mech. 16 (1967), 997-1013

121. _ Extremal polynomials associated with a system of curves in the complex plane, Adv. in Math. 3 (1969), 127-232

122. Wigert, S.: Sur les polynômes orthogonaux et l'approximation des fonctions continues, Ark. Mat. Astron. Fysik 17 (1923), no. 18 (15 pp.)

123. Zaheer, H.: Stieltjes and Van Vleck polynomials, Proc. Amer. Math. Soc. 60 (1976), 169-174

This article was processed by the author using the $\mathrm{T}_{\mathrm{E}} \mathrm{X}$ macro package from SpringerVerlag. 